\title{
Stabilization of metastable tetragonal zirconia nanocrystallites by surface modification
}

\author{
Nielsen, Mette Skovgaard; Almdal, Kristoffer; Lelieveld, A. van
}

Published in:

Journal of Materials Science

Link to article, DOI:

10.1007/s10853-010-5007-1

Publication date:

2011

Document Version

Early version, also known as pre-print

Link back to DTU Orbit

Citation (APA):

Nielsen, M. S., Almdal, K., \& Lelieveld, A. V. (2011). Stabilization of metastable tetragonal zirconia nanocrystallites by surface modification. Journal of Materials Science, 46(6), 1824-1829.

https://doi.org/10.1007/s10853-010-5007-1

\section{General rights}

Copyright and moral rights for the publications made accessible in the public portal are retained by the authors and/or other copyright owners and it is a condition of accessing publications that users recognise and abide by the legal requirements associated with these rights.

- Users may download and print one copy of any publication from the public portal for the purpose of private study or research.

- You may not further distribute the material or use it for any profit-making activity or commercial gain

- You may freely distribute the URL identifying the publication in the public portal 


\title{
Stabilization of metastable tetragonal zirconia nanocrystallites by surface modification
}

\author{
M. Skovgaard \\ DentoFit A/S, Frederiksborgvej 399, 4000 Roskilde, Denmark \\ $\&$ \\ Department of Micro- and Nanotechnology, Technical University of Denmark, \\ Frederiksborgvej 399, 4000 Roskilde, Denmark \\ ms@dentofit.com, direct: +45 467742 84, fax: +45 46774791
}

\section{K. Almdal}

Department of Micro- and Nanotechnology, Technical University of Denmark, Frederiksborgvej 399, 4000 Roskilde, Denmark

kral@nanotech.dtu.dk

\author{
A. van Lelieveld \\ DentoFit A/S, Frederiksborgvej 399, 4000 Roskilde, Denmark \\ avl@aneedle.net
}

\begin{abstract}
Metastable tetragonal zirconia nanocrystallites were studied in humid air and in water at room temperature. A stabilizing effect of different surfactants on the tetragonal phase was observed. Furthermore, the phase stability of silanized metastable tetragonal zirconia nanocrystallites was tested by prolonged boiling in water. The samples were analyzed with X-ray photoelectron spectroscopy (XPS) and x-ray diffraction (XRD). Changes in the monoclinic volume fraction in the samples were calculated. A number of surfactants were screened for their ability to stabilize the tetragonal phase upon exposure to humidity. Only silanes and phosphate esters of these were able to stabilize the tetragonal phase in water. Even as small amounts of silanes as 0.25 silane molecule per $\mathrm{nm}^{2}$ are able to stabilize the tetragonal phase in water at room temperature.

Aminopropyl trimethoxy silane and $\gamma$-methacryloxypropyl trimethoxy silane were even capable of preventing phase transformation during boiling for 48 hours in water.
\end{abstract}

Keywords: Metastable tetragonal zirconia, phase stabilization, powder, low temperature degradation. 


\section{Introduction}

Zirconia, especially the tetragonal crystal phase, has gained increasing interest, primarily because of it high strength and resistance to fracture, and its ability to catalyze organic reactions, such as isomerization of n-butane [1], synthesis of derivatives of 1,5-benzodiazepine and diaryl sulfoxides [2], and benzylation of toluene [3]. Below $1175^{\circ} \mathrm{C}$, the stable phase of zirconia is the monoclinic phase. Thus the tetragonal phase can only exist at a room temperature (RT) through stabilization. This is normally achieved by adding a dopant such as $\mathrm{Y}_{2} \mathrm{O}_{3}$ or by modifying the surface with sulfuric or phosphoric acid. It is, however, possible to obtain metastable tetragonal $\mathrm{ZrO}_{2}$ at $\mathrm{RT}$, by suitable control of the processing parameters and thereby crystal grain size. Zirconia nanocrystals can below a certain critical crystal size adopt the metastable tetragonal phase [4].

In resin composites for dental restoration zirconia particles can be added in combination with glass particles in order to render the material radiopaque (dense materials that prevent the passage of electromagnetic radiation) or to improve the mechanical properties and resistance to abrasion. Radiopacity can be useful in resin composites since it enables the distinction between composite and tooth with $\mathrm{x}$-rays. Since stabilizing the tetragonal phase with yttria, which is less radiopaque than zirconia, lowers the overall radiopacity, limitation of the dopant amount is preferable [5]. Thus dopant free stabilization of tetragonal zirconia is desirable.

During the last couple of decades the tetragonal $\rightarrow$ monoclinic $(t \rightarrow m)$ martensitic phase transformation in zirconia has been studied and the exact mechanism is still under discussion. Different mechanisms have been proposed. Most of these deal with zirconia containing stabilizing oxides such as $\mathrm{Y}_{2} \mathrm{O}_{3}$ and $\mathrm{CeO}_{2}$, and many of the proposed degradation mechanisms involve reactions with the stabilizing agent. Only limited work has been done to study the phase transformation of pure tetragonal zirconia at RT. However, some of the mechanisms based on stabilized zirconia studies are do not involve reactions with the stabilizing oxide. Sato and Shimada [6-8] based a model on reaction between water and $\mathrm{Zr}-\mathrm{O}-\mathrm{Zr}$ bonds on the surface resulting in formation of $\mathrm{OH}$-groups which in turn cause the release of the strain, which acts to stabilize the tetragonal phase. Murase and Kato [9, 10] proposed that water adsorbed on the tetragonal zirconia surface reduces the surface energy difference between the tetragonal and the monoclinic phases. This reduces the critical size of the tetragonal crystals and lead to phase transformation. However, today it is commonly accepted that the existence of metastable tetragonal zirconia at RT is due to the stabilizing effect of oxygen vacancies in the crystal lattice. Yoshimuru et al [11,12] proposed that phase transformation is a result of adsorption of water on the surface leading to formation of $\mathrm{Zr}-\mathrm{OH}$ and/or $\mathrm{Y}-\mathrm{OH}$ which creates stressed sites on the surface. This mechanism is based on the formation of strain in the surface and lattice, caused by occupation of oxygen vacancies by $\mathrm{OH}^{-}$ions, nucleating monoclinic phase in the tetragonal crystallites. Kim et al [13] proposed a mechanism based on $\mathrm{OH}^{-}$ions diffusing through oxygen vacancies and reacting with these under formation of $\mathrm{Zr}-\mathrm{OH}$ bonds. This reaction leads to a build-up of tensile strain, which will induce the phase transformation. Guo [14-16] proposed a mechanism consisting of the following steps: 1) water is absorbed on the surface, 2) $\mathrm{OH}^{-}$is formed by reaction of water with $\mathrm{O}^{2-}$ on the zirconia surface, 3) diffusion of $\mathrm{OH}^{-}$, 4) formation of proton defects by filling of oxygen vacancies with hydroxyl, and 5) $t \rightarrow m$ phase 
transformation when the concentration of oxygen vacancies is reduced to the extent that the tetragonal phase is no longer stabilized.

Different types of surface modifying agents such as trimethoxy silanes and phosphate esters are used to secure a strong bond between the filler surface and the polymer matrix in dentistry. The present study investigates the effect of surface modification with different surface modifying agents, especially silanes, on the phase stability of metastable tetragonal zirconia crystals in humid atmosphere and in water. Furthermore the stability of the surface modified tetragonal crystals in boiling water is also investigated.

\section{Experimental}

All chemicals were supplied by Sigma-Aldrich (St. Louis, MO, USA) and they were all used as received. Highly porous nanocrystalline tetragonal zirconia powders were synthesized as previously described [17] by controlled hydrolysis of $\mathrm{ZrOCl}_{2}$ followed by careful calcination. The tetragonal zirconia powder is extremely porous and has specific surface area of $\sim 150 \mathrm{~m}^{2} / \mathrm{g}$. The synthesized tetragonal zirconia powders were kept in water free environment for further treatment.

\section{Surface modification of zirconia powder}

In inert atmosphere in a glovebox, $7 \mathrm{~g}$ zirconia powder was stirred with $40 \mathrm{ml}$ anhydrous methanol and $7 \mathrm{ml}$ of surfactant overnight. The surface modified particles were filtered and washed three times with anhydrous methanol. The samples were exposed to air and analyzed with x-ray diffraction (XRD). For each sample, parts of the modified zirconia was mixed in the glovebox with a dimethacrylic monomer mixture consisting of bisphenol-A diglycidyl ether dimethacrylate (Bis-GMA), urethane dimethacrylate (UDMA) and triethylene glycol dimethacrylate (TEGDMA) in the ratio (36/44/ $20 \mathrm{wt} \%$ ) in combination with a photo-polymerization system composed of camphorquinone and ethyl 4dimethylamino benzoate both in a content of $0.5 \mathrm{wt}-\%$. A sample was placed between two glass plates and cured on each side for 2 min using blue light $\left(1100 \mathrm{~mW} / \mathrm{cm}^{2}\right)$ from a Bluephase ${ }^{\circledR}$ light probe (Ivoclar Vivadent). Such samples are termed matrix dispersed zirconia. After curing, the resulting composites were stored at $37^{\circ} \mathrm{C}$ in water for 2-30 days and subsequently subjected to XRD analysis.

\section{Stability test in air}

$1 \mathrm{~g}$ zirconia powder was stirred with $10 \mathrm{ml}$ anhydrous methanol and $\gamma$ methacryloxypropyl trimethoxy silane (MPTMS) in amounts ranging from 0 $1000 \mu \mathrm{L}$ in dry air in a glove box. After 2 hours the mixtures were filtered and washed twice with anhydrous methanol to remove unreacted silane and dried inside the glove box. The stability was tested by exposing the samples for XRD to ambient atmosphere prior to the analysis. A reference samples for each sample were prepare by mixing the modified samples with resin and cure as described above. The matrix dispersed zirconia samples were after curing analyzed with XRD. 


\section{Stability test in water}

For the stability test $1 \mathrm{~g}$ zirconia powder was stirred with $10 \mathrm{ml}$ anhydrous methanol and aminopropyl trimethoxy silane (APTMS) in amounts ranging from $0-2000 \mu \mathrm{L}$ in dry air in a glove box. After 2 hours the mixtures were filtered and washed twice with anhydrous methanol to remove unreacted silane and dried inside the glove box. The stability was tested by adding a few drops of water to the samples for XRD analysis. A reference sample for each sample was prepared by mixing the modified sample with resin and cure as described above. The matrix dispersed zirconia samples were analyzed with XRD after curing.

\section{Stability in boiling water}

$15 \mathrm{~g}$ zirconia powder was stirred with $100 \mathrm{ml}$ anhydrous methanol and $10 \mathrm{ml}$ of either APTMS or MPTMS for 2 hours in inert atmosphere in a glovebox. The mixture was filtered and the zirconia was washed twice with anhydrous methanol, $150 \mathrm{ml}$ in total. The silanized zirconia samples were dried at RT in the glovebox. The silanized samples were then boiled in $400 \mathrm{ml}$ water and samples were collected, filtered and dried at RT. After drying, the samples were subjected to XRD analysis. Samples were collected after 1, 2, 4, 8, 24 and 48 hours of boiling. $100 \mathrm{ml}$ water was added after the first $8 \mathrm{~h}$ to prevent mixture from drying or burn.

\section{Characterization}

\section{$X$-Ray Diffraction $(X R D)$ evaluation}

XRD patterns were scanned in 0.1 steps $(2 \theta)$, in the $2 \theta$ range from $20^{\circ}$ to $65^{\circ}$, with a fixed counting time (30 sec.). The XRD patterns were analyzed using $\mathrm{WinX}^{\mathrm{POW}}$ software. The tetragonal and monoclinic volume fractions $\left(\mathrm{v}_{\mathrm{t}}\right.$ and $\left.\mathrm{v}_{\mathrm{m}}\right)$ were calculated from the integral intensities of the monoclinic diffraction lines $(-1$ $\left.\begin{array}{ll}1 & 1\end{array}\right)$ and $\left(\begin{array}{lll}1 & 1 & 1\end{array}\right)$ and the tetragonal diffraction line $\left(\begin{array}{lll}1 & 0 & 1\end{array}\right)$, following a procedure proposed by Toraya et al [18].

\section{$X$-ray photoelectron spectroscopy (XPS) evaluation}

Surface analyses were performed with a K-alpha monochromated, XPS spectrometer from Thermo Fisher Scientific Inc., Waltham, MA United States.

\section{Surface Area Measurement}

The specific surface area of the zirconia powder analysis was done with $\mathrm{N}_{2}$ adsorption for BET (Brunauer-Emmett-Teller) determination (Autosorb-AS6, Quantachrome, Boynton Beach, FL). A number of pure zirconia samples were analyzed the specific surface area was determent to $\approx 150 \mathrm{~m}^{2} / \mathrm{g}$. 


\section{Results}

\section{Surface modification and phase transformation}

None of the analyzed samples phase transformed as a result of exposure to humidity in the air. A number of different surfactant classes were tested but only silanes and phosphates stabilize the tetragonal phase when exposed to water. All the tested surfactants were able to stabilize the tetragonal phase when exposed to humidity in the air (Table 1).

Fig. 1 shows the XRD patterns of a silanized and an unmodified zirconia samples, respectively, exposed to humidity in air. The silanized zirconia exhibit a trace characteristic of the tetragonal phase (curve a) whereas the unmodified zirconia trace is dominated by monoclinic reflections (curve b). Thus, unmodified zirconia goes through the $\mathrm{t} \rightarrow \mathrm{m}$ transition after brief exposure to ambient atmosphere, whereas the tetragonal phase in silanized zirconia is stable in humid air. No phase transformation is observed even after a week of exposure. The same stability is seen with silanized tetragonal zirconia when cured in resin and stored in water at $37{ }^{\circ} \mathrm{C}$ for 30 days. Under these conditions the untreated zirconia phase transforms in less than 2 days (not shown).

\section{Stability in air and water}

In order to test how small amounts of silane are sufficient to hinder phase transformation of zirconia particles exposed to ambient atmosphere a set of zirconia particles with decreasing amount of silanization was prepared through treatment of particles with increasingly diluted solution of silanization agent. The lowest silane coverages were not detectable in XPS. It is chosen to use the theoretical number of silane molecules per $\mathrm{nm}^{2}$ (assuming $100 \%$ conversion) and not the actual number when comparing data. This means the actual number is potentially lower as unreacted silanization agent in the reaction mixture is washed out during the silanization. The results are plotted inFig. 2. It can be seen that even very small amounts of MPTMS are sufficient to achieve stabilization of the tetragonal phase. When the theoretical number of silane molecules per $\mathrm{nm}^{2}$ gets down to 0.35 the volume fraction of monoclinic zirconia starts to increase and at coverage degree of $\sim 0.017$ the phase transformation reaches a $\mathrm{v}_{\mathrm{m}}$ of $\sim 0.6$. This is close to, but not equal to the level of unmodified zirconia, which reaches a $\mathrm{v}_{\mathrm{m}}$ of $\sim 0.7$ upon exposure to the ambient atmosphere.

The same trend is observed for zirconia silanized with APTMS (Fig. 3) and exposed to water at RT. Below a surface coverage of 0.25 silane molecule per $\mathrm{nm}^{2}$ the volume fraction of monoclinic zirconia starts to increase and at the coverage of 0.008 silane molecules per $\mathrm{nm}^{2}$ the volume fraction reaches 0.63 . Even this minute surfactant coverage prevent phase transformation to the same extend as the unmodified reference sample, which becomes almost entirely monoclinic after exposure to water [19]. The curve asymptotically approaches a monoclinic volume fraction of $\sim 0.3$. This level is as supported by XRD analysis of the reference samples due to phase transformation coursed by the silanization. Similar to the MPTMS experiments, the theoretical number of silane molecules per $\mathrm{nm}^{2}$ is used as variable due to limitations in XPS. 
The XRD patterns (illustrated in Fig. 4 and Fig. 5) of the boiled samples show the stability of the tetragonal phase in the silanized samples. $48 \mathrm{~h}$ of boiling in water is insufficient to induce change in the monoclinic volume fraction in both of the samples. A small increase in the monoclinic phase occurs within the first hour. In XPS the atom- $\%$ of silicon were measured to $2-2.5 \%$ for both samples prior to the boiling test. No silane was found on unmodified zirconia samples.

\section{Discussion}

The results from the study of the ability of different surfactants to stabilize zirconia are summarized in table 1 . It is observed that all the tested surfactants are able to stabilize the tetragonal phase on exposure to humidity in the air. But only silanes and phosphates were able to stabilize the tetragonal phase enough to withstand exposure to water, when aged in a di-methacrylate matrix. This is in accordance with the fact that silanes are the only tested surfactant forming covalent bonds to the surface and phosphate esters are known to bind zirconia strongly [20]. This means that the bonds between the zirconia surface and either the silane and the phosphate are strong enough to withstand hydrolysis. The necessity of testing the water stability of the zirconia crystal phase in a polymer matrix is due to the fact that the surface modified powder is hydrophobic to the extent that mixing with water is difficult thus hindering the access of water to the zirconia surface, whereas in the polymer matrix the water is forced to the surface as the polymer absorbs water. The polymer almost works as a surfactant facilitating wetting of the zirconia surface.

The powder is very sensitive to water vapor and few seconds of air exposure is sufficient to induce the martensitic $\mathrm{t} \rightarrow \mathrm{m}$ phase transformation [21]. This can be observed in Fig. 1, which shows x-ray patterns of pure tetragonal zirconia that is exposed to humid air and undergoes martensitic phase transformation resulting in monoclinic crystals. The silanization prevents the transformation probably due to the produced hydrophobic surface that hinders the access of water molecules to the surface. Furthermore surface treated zirconia floats on water where untreated zirconia disperses. Alternatively the stabilization of the tetragonal phase can also be a result of a change in the surface energy which increases the activation energy of the phase transformation. The initial amount of monoclinic zirconia in the sample is most likely due to small amounts of water in the methanol used for the silanization or other contamination with water.

Samples silanized with APTMS show a lower reactivity than MPTMS silanized samples during stability tests in air and water in spite of the fact that the APTMS modified samples are tested in water and as such are expected to have a higher monoclinic volume fraction. The differences in sensitivity of samples with the two different silane modifications are probably caused by a higher reactivity of APTMS due to the autocatalytic catalytically effect of the primary amine in the silanization reaction [22]. This will give a higher actual coverage of the APTMS modified zirconia powder than for the MPTMS modified zirconia powder leading to a lower sensitivity towards humidity.

The results of the boiling experiment show that the $\mathrm{Si}-\mathrm{O}-\mathrm{Zr}$ bonds are sufficiently stable that the surface bound silanes prevent the $t \rightarrow m$ zirconia phase 
transformation even after 48 hours in boiling water. The small initial phase transformation, observed in both Fig. 4 and Fig. 5, can be due to either a small number of unmodified zirconia crystals or crystals with very low silane densities. Such crystals will upon water contact rapidly transform into the monoclinic phase. The monoclinic volume fraction is stabilized at 0.5 after 2 hours for the APTMS modified sample and somewhat slower for the MPTMS modified sample. The silanized zirconia is very hydrophobic preventing wetting with water. However, boiling the silanized zirconia in water for a short time overcomes this resistance and the mixing with water ensues. This takes it little longer for the MPTMS modified samples. As expected the MPTMS modified zirconia is powder more hydrophobic than the APTMS modified. This difference in hydrophilicity is probably the reason for the lower transformation rate in the MPTMS modified samples.

The generally accepted models for the stability of metastability of tetragonal zirconia and the induction of phase transformation to monoclinic zirconia all involve interaction of absorbed water on the surface with oxygen vacancies to form proton defects in the crystal structure. In the models, the water absorption is followed by formation of $\mathrm{OH}^{-}$that either stays in the surface or diffuses into the crystal. Thus from a surface point of view, it is the absorption of water that is the critical step. The observed stability of tetragonal zirconia surface modified with either silanes or phosphate esters cannot be reconciled with these models. The amount of e.g. silanization required to obtain stabilization is far from enough to hinder absorption of water since at a silane density of 0.25 molecules per $\mathrm{nm}^{2}$ only $10 \%$ of the possible sites are covered (determined as - $\mathrm{OH}$ group by titration with $\mathrm{LiAlH}_{4}$ ). We hypothesize that the surface modification blocks the access of water to certain active sites - of presently unknown nature - which are crucial in the phase transformation mechanism. It remains a possibility, however, that the silanization reduces the surface energy more for the tetragonal phase than for the monoclinic phase and thereby shifts the critical crystal size upwards. Our data do not permit distinguishing between these two models.

\section{Conclusion}

Within the group of the screened surfactants surface modification with phosphates and silanes stabilize tetragonal zirconia in water. Even as small amounts of silane as 0.25 silane molecules per $\mathrm{nm}^{2}$ are enough to stabilize the tetragonal phase in water at room temperature and the stabilizing properties of silanes are even sufficient to withstand boiling in water for 48 hours.

\section{Acknowledgement}

Financially support from DentoFit A/S is gratefully acknowledged. We thank Mads Gudik-

Sørensen for providing zirconia samples.

\section{References}


[2] Reddy BM, Sreekanth PM, Lakshmanan P (2005) Journal of Molecular Catalysis A-Chemical 237:93

[3] Mishra MK, Tyagi B, Jasra RV (2004) J Mol Catal A-Chem 223:61

[4] Becker J, Hald P, Bremholm M et al (2008) ACS Nano 2:1058

[5] Zhang X, Kolb BU, Hanggi DA et al (2001) WO 01/30305 A1

[6] Sato T, Ohtaki S, Endo T et al (1985) J Am Ceram Soc 68:C320

[7] Sato T, Shimada M (1985) J Mater Sci 20:3988

[8] Sato T, Shimada M (1985) American Ceramic Society Bulletin 64:1382

[9] Murase Y, Kato E (1983) J Am Ceram Soc 66:196

[10] Murase Y, Kato E (1979) J Am Ceram Soc 62:527

[11] Yoshimura M (1988) American Ceramic Society Bulletin 67:1950

[12] Yoshimura M, Noma T, Kawabata K et al (1987) J Mater Sci Lett 6:465

[13] Kim YS, Jung CH, Park JY (1994) J Nucl Mater 209:326

[14] Guo X (2001) J Mater Sci 36:3737

[15] Guo X (1999) Journal of Physics and Chemistry of Solids 60:539

[16] Guo X (1998) Solid State Ionics 112:113

[17] Skovgaard M, Ahniyaz A, Sørensen BF, et al (2010). J Eur Ceram Soc, 30:2749-2755.

[18] Toraya H, Yoshimura M, Somiya S (1984) J Am Ceram Soc 67:C119

[19] Skovgaard M, Almdal K, van Lelieveld A (2010) J Mater Sci (online)

[20] Gao W, Dickinson L, Grozinger C et al (1996) Langmuir 12:6429

[21] Van Lelieveld A, Nielsen MS, Almdal K et al EP1996144-A2, US2010016465-A1

[22] Blitz JP, Murthy RSS, Leyden DE (1987) J Am Chem Soc 109:7141

\section{Tables}

Table 1 Zirconia surface modified with different groups of surfactants tested for its ability to phase transform (PT) in air and in water (matrix dispersed zirconia)

\begin{tabular}{|c|c|c|c|}
\hline $\begin{array}{l}\text { Group of } \\
\text { surfactants }\end{array}$ & Name & PT in air & $\begin{array}{c}\text { PT in water } \\
\text { (matrix dispersed) }\end{array}$ \\
\hline \multirow[t]{3}{*}{ Carboxylic acids } & Hexylic acid & no & yes \\
\hline & $2-[2-(2-$ & NA & yes \\
\hline & Methoxyethoxy)ethoxy]acetic acid & & \\
\hline Sulfonic acid & 4-Dodecylbenzenesulfonic acid & no & yes \\
\hline Phosphate ester & $\begin{array}{l}\text { Ethylene glycol methacrylate } \\
\text { phosphate }\end{array}$ & no & no \\
\hline \multirow[t]{3}{*}{ Silanes } & $\begin{array}{l}\gamma \text {-Methacryloxypropyl trimethoxy } \\
\text { silane (MPTMS) }\end{array}$ & no & no \\
\hline & $\begin{array}{l}\text { Aminopropyl trimethoxy silane } \\
\text { (APTMS) }\end{array}$ & no & no \\
\hline & Methyl trimethoxy silane & no & no \\
\hline \multirow[t]{2}{*}{ Amines } & $\begin{array}{l}\text { O-(2-aminopropyl)-ó-( } 2 \text { - } \\
\text { methoxyethyl) polypropylenglycole } \\
500\end{array}$ & NA & yes \\
\hline & Tetrabutyl ammonium bromide & NA & yes \\
\hline
\end{tabular}




\section{Figure captions}

Fig. 1 XRD patterns for zirconia silanized with MPTMS exposed to air (a) and unmodified zirconia exposed to air (b) $\mathrm{t}=$ tetragonal reflection, $\mathrm{m}=$ monoclinic reflection.

Fig. 3 Volume fraction of monoclinic zirconia in MPTMS modified samples in ambient atmosphere as a function of the theoretical number of silane molecules per $\mathrm{nm}^{2}$

Fig. 4 Volume fraction of monoclinic zirconia in APTMS modified samples exposed to water as a function of the theoretical number of silane molecules per $\mathrm{nm}^{2}$

Fig. 5 XRD patterns of APTMS modified zirconia boiled for 0 (dashed black), 1 (solid blue), 2 (dotted purple) and 48 hours (green dash dot)

Fig. 6 XRD patterns of MPTMS modified zirconia boiled of 0 (dashed black), 1 (solid blue), 2 (dotted purple) and 48 hours (green dash dot)

\section{Figures}

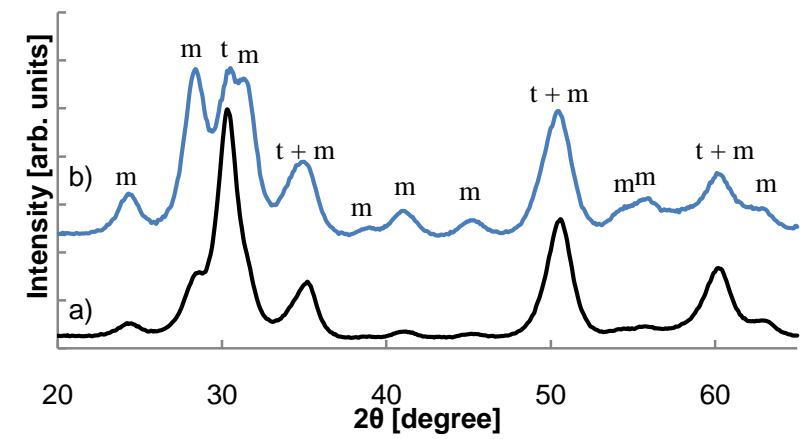

Fig. 2 XRD patterns for zirconia silanized with MPTMS exposed to air (a) and unmodified zirconia exposed to air (b) $\mathrm{t}=$ tetragonal reflection, $\mathrm{m}=$ monoclinic reflection.

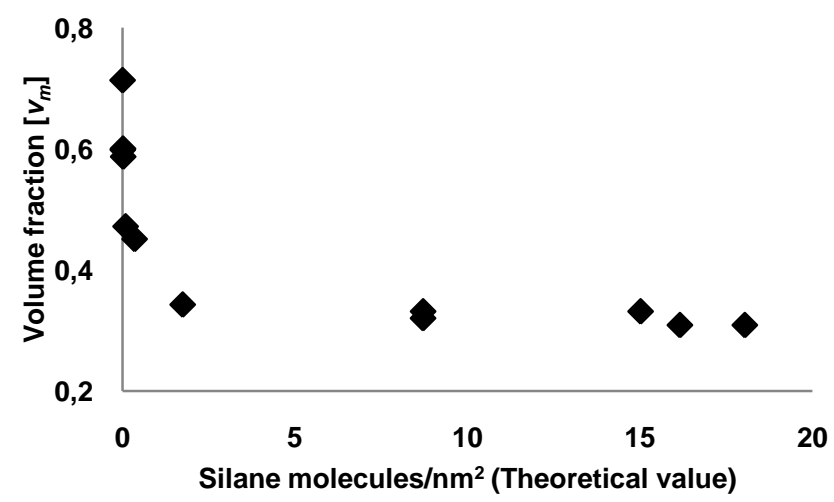

Fig. 3 Volume fraction of monoclinic zirconia in MPTMS modified samples in ambient atmosphere as a function of the theoretical number of silane molecules per $\mathrm{nm}^{2}$ 


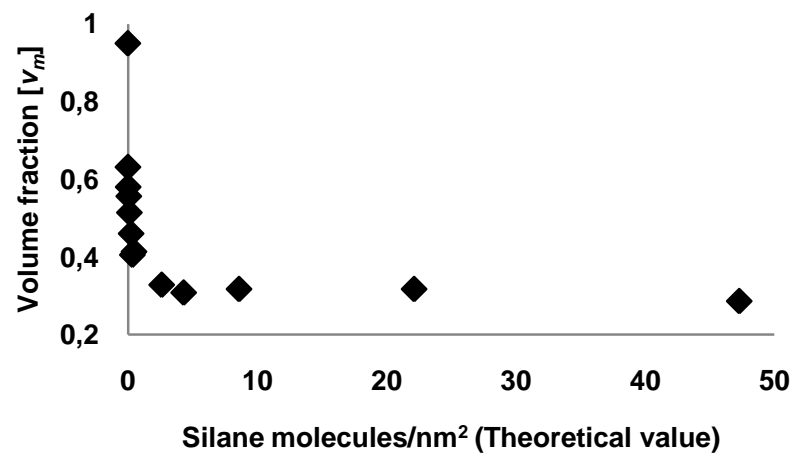

Fig. 4 Volume fraction of monoclinic zirconia in APTMS modified samples exposed to water as a function of the theoretical number of silane molecules per $\mathrm{nm}^{2}$

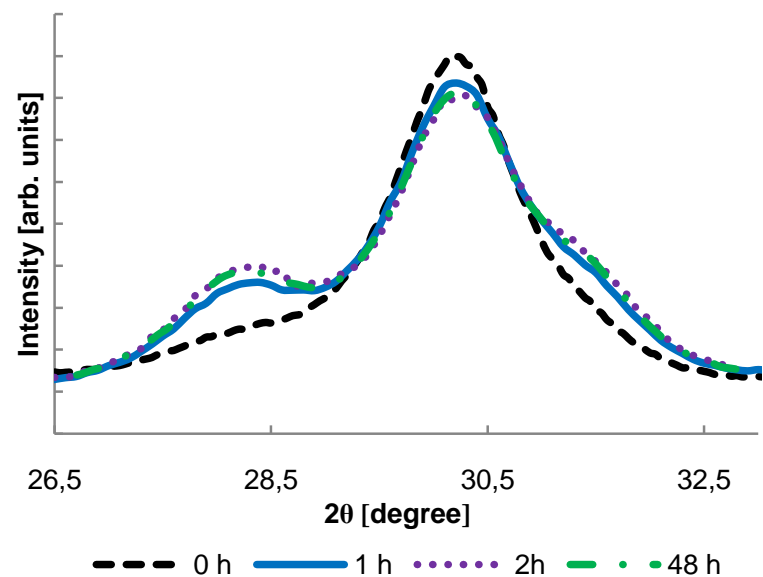

Fig. 5 XRD patterns of APTMS modified zirconia boiled for 0 (dashed black), 1 (solid blue), 2 (dotted purple) and 48 hours (green dash dot)

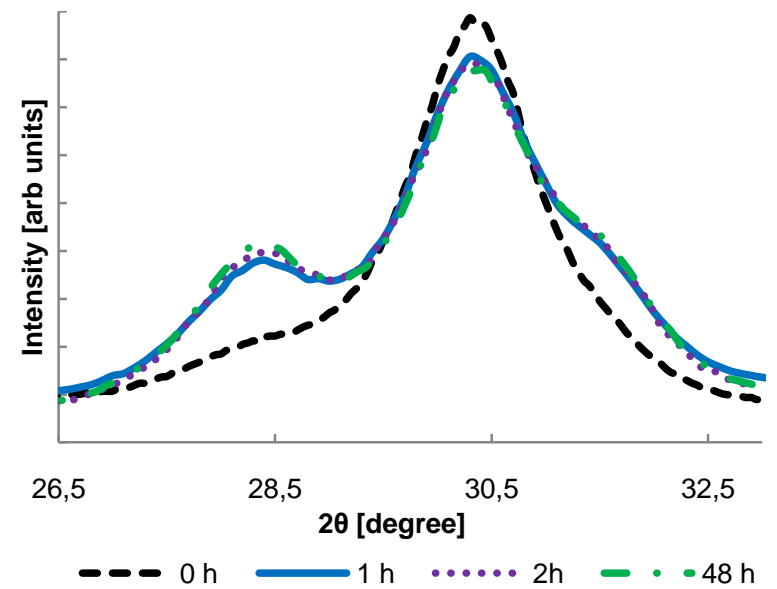

Fig. 6 XRD patterns of MPTMS modified zirconia boiled of 0 (dashed black), 1 (solid blue), 2 (dotted purple) and 48 hours (green dash dot) 